\title{
Effect of sputtering conditions on the gas sensitivity of copper oxide thin films
}

\author{
P. Samarasekara* and N. U. S. Yapa \\ Department of Physics, University of Ruhuna, Matara, Sri Lanka
}

\begin{abstract}
Copper Oxide (CuO) thin films were deposited on conductive glass substrates using DC reactive sputtering in a mixture of Argon and Oxygen gases. The sputtering pressure and the substrate temperature were increased from 6 to 8.5 mbar and from 70 to $192{ }^{\circ} \mathrm{C}$, respectively. All the synthesized films contain single phase of $\mathrm{CuO}$ in this range of pressure and substrate temperature. According to Scherrer formula, the crystallite sizes vary from 9.03 to $22.47 \mathrm{~nm}$ as sputtering pressure is varied from 8.5 to 6 mbar. The crystallites favoring perpendicular orientations dominate at higher deposition pressures due to higher deposition rates. Due to smaller crystallite sizes, the film deposited at $192{ }^{\circ} \mathrm{C}$ under 8.5 mbar pressure provides a gas sensitivity as high as 19.26 after keeping10 minutes in $\mathrm{CO}_{2}$ gas at room temperature. The sample fabricated at $192{ }^{\circ} \mathrm{C}$ and 9 mbar indicates a sharp drop of $\mathrm{CO}_{2}$ gas sensitivity from 3.67 to 0.84 at operating temperature $75{ }^{\circ} \mathrm{C}$. All theses samples are not sensitive to $\mathrm{N}_{2}$ gas according to cross-sensitivity measured in $\mathrm{N}_{2}$ gas.
\end{abstract}

\section{INTRODUCTION}

Thin films of $\mathrm{CuO}$ find some potential applications in solar energy converting devices, electro-chromic devices, transparent conducting devices, gas sensors [1-4] and superconducting devices [5]. Annealing effects on the properties of Copper oxide thin films prepared by chemical deposition have been studied [6]. Thin films of $\mathrm{CuO}$ have been previously synthesized using molecular-beam-epitaxy method [7], sol-gel technique [8].

\footnotetext{
*Corresponding author: pubudus@phy.ruh.ac.lk
} 
According to some early reports, it is difficult to crystallize the single phase of $\mathrm{CuO}$ [4]. The deposition methods in high vacuum such as sputtering provide films with high purity. Earlier we have deposited thin films of zinc oxide and copper oxide for photo-voltaic applications $[9,10]$. The properties of sputtered copper oxide films mainly depend on the deposition temperature and the sputtering pressure. According to some of our early studies, the films of magnetic materials could be crystallized only above some minimum crystallization temperature $[11,12]$.

Because the existence of too much $\mathrm{CO}_{2}$ gas is harmful to humans, the gas sensitivity was investigated in $\mathrm{CO}_{2}$ gas. Also most of the oxide materials including $\mathrm{CuO}$ are chemically stable against environmental corrosion. Previously, the variation of $\mathrm{CO}_{2}$ gas sensitivity with temperature has been investigated by us for $\mathrm{CuO}$ thin films. In this report, the variation of film properties with sputtering conditions and the variation of gas sensitivity with time will be explained as well. The orientation of different types of $\mathrm{CuO}$ particles in thin film changes with sputtering pressure. Investigation of the relationship between gas sensitivity and the particle size of $\mathrm{CuO}$ thin films was the main purpose of this project. Especially in miniaturized equipment used in spacecrafts and other compact devices, thin films of materials are useful to determine the existence of some gases.

\section{EXPERIMENT}

Films were synthesized in a vacuum chamber with a base pressure of 4.2 mbar by means of the Edwards S150B sputter coater. All the $\mathrm{CuO}$ films were deposited on the conductive surface of cleaned ITO conductive glass substrates with area $2 \mathrm{~cm} \times 2 \mathrm{~cm}$ for 6 hours using a circular copper target with diameter $6 \mathrm{~cm}$. The separation between the target and the substrate was approximately $1.5 \mathrm{~cm}$. Argon and oxygen gases served as sputtering and reactive gases, respectively in this reactive sputtering process. The argon gas was introduced into the chamber through a flow controller with fine adjustments. The oxygen content in the residual amount of air in the chamber was enough to crystallize the oxide phase in thin films. The total pressure of $\mathrm{Ar} / \mathrm{O}_{2}$ mixture was increased from 6 to 8.5 mbar by raising the rate of Argon flow entering into the chamber in this dynamic sputtering process. A heater coil attached to an external power supply was used to heat the substrate, and substrate temperature varied from $70{ }^{0} \mathrm{C}$ to $192{ }^{\circ} \mathrm{C}$, was measured by means of a thermocouple coupled to a digital thermometer. The substrate temperature and the 
sputtering pressure could not be raised above $150{ }^{\circ} \mathrm{C}$ and 8 mbar, respectively, due to the over heating of the vacuum chamber. The DC current and DC voltage of the sputtering power supply varied from 5 to $35 \mathrm{~mA}$ and 0.4 to $1.9 \mathrm{kV}$, respectively depending on the deposition pressure.

The structure of the synthesized films was determined using X-ray diffraction patterns (XRD). The resistance of $\mathrm{CuO}$ films located in a glass tube was measured in a continuous $\mathrm{CO}_{2}$ gas flow, in pure $\mathrm{N}_{2}$ and in air with temperature and time. The bulk resistance of the sample was measured using high mega ohm Leader LCR745 meter. Because the same sample clipped at same points was measured in all three gases, the length and the cross sectional are of the sample remained unchanged. Therefore, the ratio between two resistances directly gives the ratio between two resistivities. The temperature was varied from 30 to $200{ }^{0} \mathrm{C}$ using a heater coil, and the corresponding temperature was measured using a digital thermocouple attached to the sample. The bulk resistivity of conductive glass is $0.13 \mathrm{ohm} \mathrm{m}$.

\section{RESULTS AND DISCUSSION}

The XRD pattern of $\mathrm{CuO}$ film deposited at $192^{\circ} \mathrm{C}$ and 8.5 mbar total pressure is given in Fig. 1. The film indicates a strong in-plane (020) orientation. All the XRD peaks in these patterns belong to the powder diffraction pattern of $\mathrm{CuO}$ implying that the single phase of $\mathrm{CuO}$ can be crystallized in the substrate temperature range of $151-192{ }^{0} \mathrm{C}$ and pressure range 6-8.5 mbar. The XRD pattern of conductive side of substrate was measured, and conductive glass substrate did not contribute to the XRD pattern in this range of $2 \theta$.

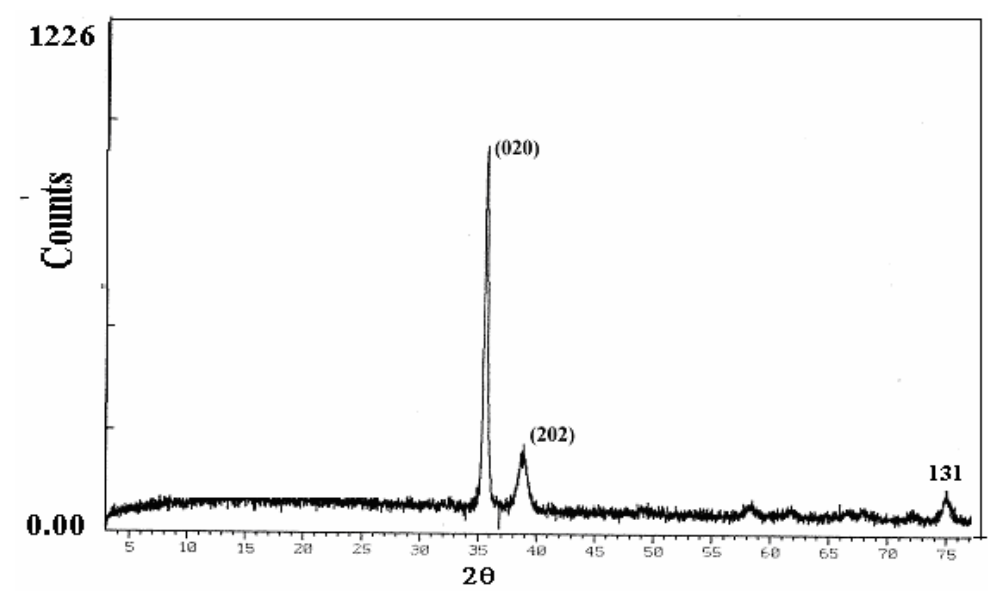

Fig 1. XRD pattern of film deposited at $192^{\circ} \mathrm{C}$ and $8.5 \mathrm{mbar}$ 
The crystallite sizes were estimated using Scherrer formula for the two peaks at $\theta=17.8^{0}$ and $19.35^{\circ}$ in XRD patterns. The graph of crystallite sizes estimated for peaks at $\theta=17.8^{0}$ (solid line) and $\theta=19.35^{\circ}$ (dotted line) versus total sputtering pressure for the films deposited at $192{ }^{0} \mathrm{C}$ for 6 hours is shown in Fig. 2. According to this graph, the volume of crystalline favoring the in-plane orientation gradually decreases with the sputtering pressure. The $c / a$ ratio of $\mathrm{CuO}$ cell (where $a$ and $c$ are the lattice constants of $\mathrm{CuO}$ unit cell) is greater than one, and therefore the perpendicular and in-plane orientations can be textured at higher and smaller deposition rates, respectively[13]. The Argon pressure inside the sputter coater is given by the difference between the total pressure and the base pressure. Raising the Argon pressure increases the deposition rate, and higher deposition rates favor the growth of perpendicularly oriented grains. Because $\mathrm{C}$-axis of $\mathrm{CuO}$ cell is longer than the length of other two axes, the long axis (C-axis) orients in a direction perpendicular to the film plane.

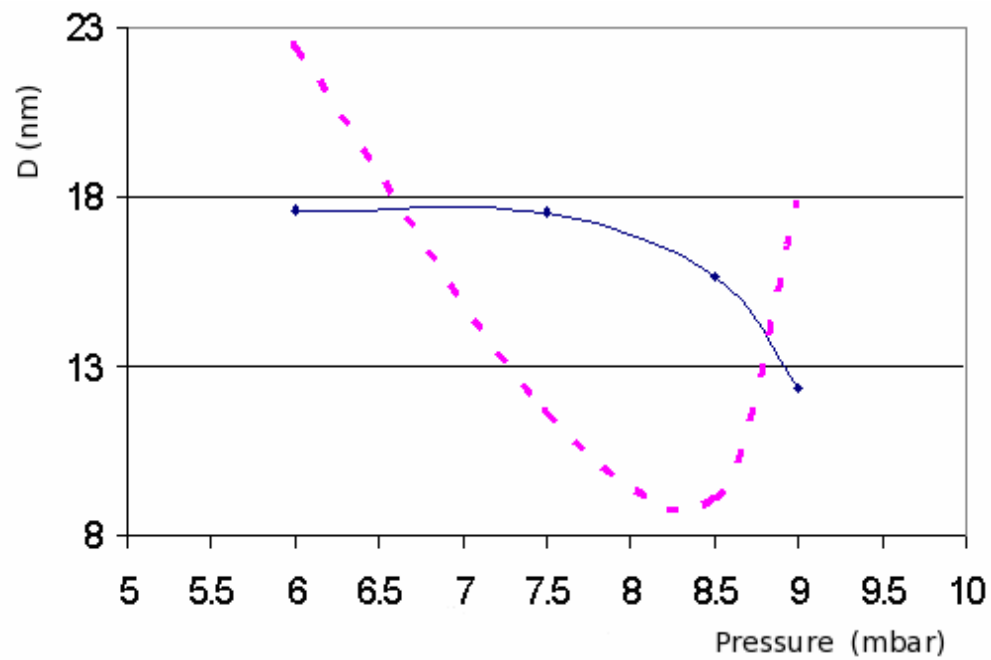

Fig. 2. Plot of crystallite sizes estimated for peaks at $\theta=17.8^{0}$ (solid line) and $\theta=19.35^{0}$ (dotted line) versus total sputtering pressure

The gas sensitivity ( $\left.S=\rho_{\text {gas }} / \rho_{\text {air }}\right)$ ) is defined as the ratio of resistivity measured at particular gas $\left(\rho_{\text {gas }}\right)$ to that of air $\left(\rho_{\text {air }}\right)$ [1-3]. Graphs between the sensitivity measured at $\mathrm{CO}_{2}$ gas and time for $\mathrm{CuO}$ films sputtered at $192{ }^{0} \mathrm{C}$ under 9 (thick solid line), 7.5 (thin solid line) and 6 mbar (dashed line) pressures are shown in Fig. 3. In this case, the sensitivity was measured at room temperature in order to investigate the variation with time. According to this graph the sensitivity gradually increases with time. It saturates within the first ten minutes for sample deposited at 9 mbar. This implies that the sensitivity increases with the amount of absorbed gas. The sensitivity at room temperature versus time measured at $\mathrm{CO}_{2}$ (solid line) 
and $\mathrm{N}_{2}$ (dashed line) gases for $\mathrm{CuO}$ films sputtered at $192{ }^{\circ} \mathrm{C}$ under 8.5 mbar pressure is given in Fig. 4. This sample indicated the highest sensitivity after being kept in $\mathrm{CO}_{2}$ gas for 10 minutes. According to Fig. 2, the crystallite size estimated using the (202) peak indicates a minimum for the sample deposited at $192{ }^{0} \mathrm{C} 8.5 \mathrm{mbar}$. Because smaller particle sizes always provide larger surface areas, the maximum gas sensitivity can be observed for samples with smallest crystallite sizes. The gas sensitivity measured in $\mathrm{N}_{2}$ gas for the samples given in Fig. 3 also did not vary with time similar to that of sample described in Fig. 4. Therefore the cross sensitivity of those samples were not given in Fig. 3.

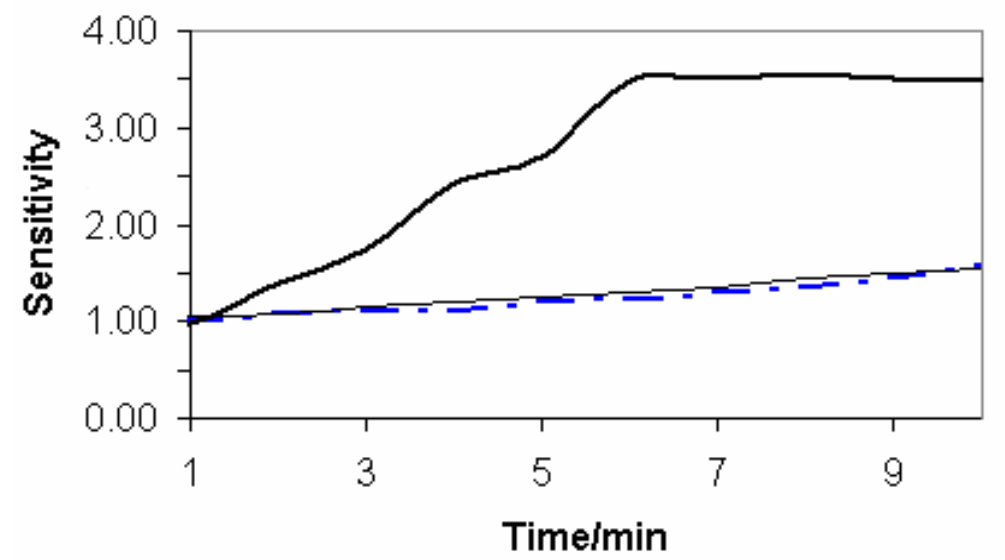

Fig. 3. Sensitivity at room temperature versus time for $\mathrm{CuO}$ films sputtered at $192{ }^{0} \mathrm{C}$ under 9 (thick solid line), 7.5 (thin solid line) and 6 mbar (dashed line) pressures

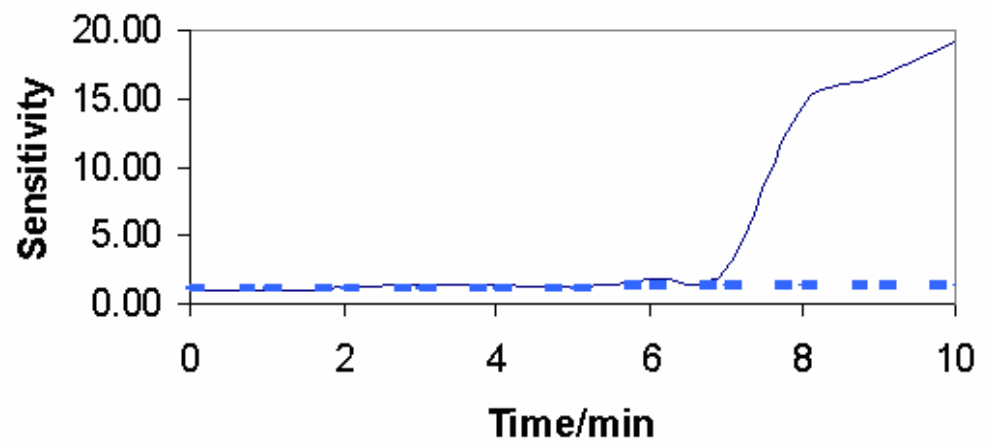

Fig. 4. Sensitivity at room temperature in $\mathrm{CO}_{2}$ (solid line) and $\mathrm{N}_{2}$ gases (dashed line) versus time for $\mathrm{CuO}$ films sputtered at $192{ }^{0} \mathrm{C}$ under 8.5 mbar pressure

The Fig. 5 indicates the sensitivity measured at $\mathrm{CO}_{2}$ (solid line) and $\mathrm{N}_{2}$ (dashed line) gases versus temperature for the $\mathrm{CuO}$ film fabricated at $192{ }^{\circ} \mathrm{C}$ and 9 mbar. The sensitivity in $\mathrm{CO}_{2}$ was measured with temperature after the sensitivity reached the saturated value at room temperature. The sensitivity in $\mathrm{CO}_{2}$ suddenly drops to a lowest value at $75{ }^{0} \mathrm{C}$. The smaller the resistivity is the lager the conductivity. Therefore, this sample can be used as a gas sensor at the operating temperature of $75{ }^{\circ} \mathrm{C}$. Although the sample grown at $192{ }^{0} \mathrm{C}$ under 8.5 mbar shows highest sensitivity at room temperature within 10 minutes, the 
sensitivity of this sample can not be measured with temperature due to the limitation of our instruments. Altering the underlying charge carriers in the Copper oxide film or the electrical conduction between individual crystal grains in the film due to surface adsorption may be a possible reason for this high gas sensitivity.

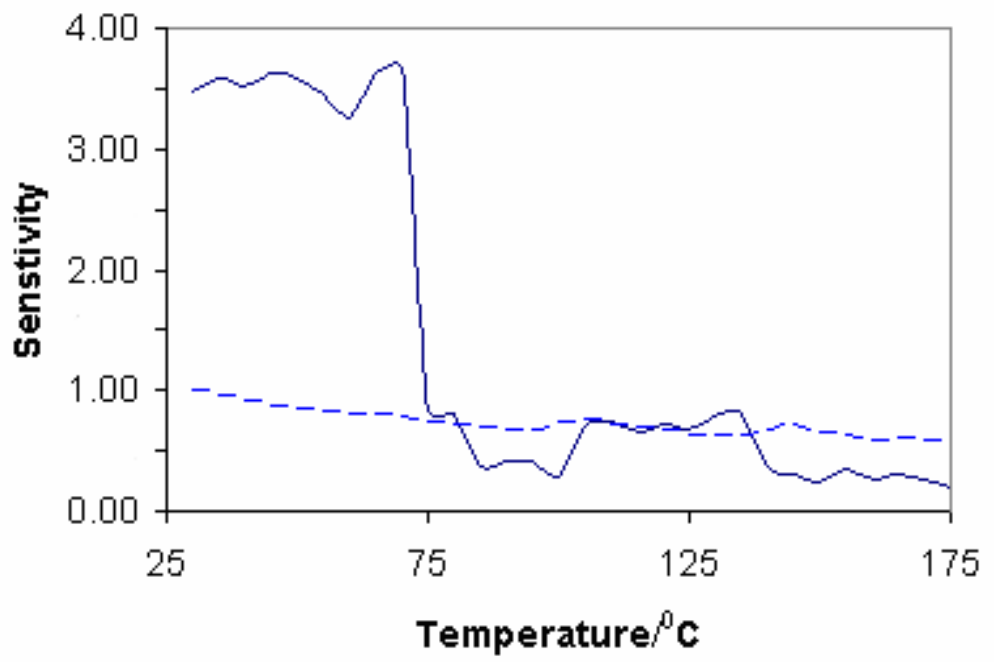

Fig. 5. Graph of sensitivity measured in $\mathrm{CO}_{2}$ (solid line) and $\mathrm{N}_{2}$ (dashed line) gases versus temperature for the $\mathrm{CuO}$ film fabricated at $192{ }^{\circ} \mathrm{C}$ and 9 mbar.

\section{CONCLUSIONS}

$\mathrm{CuO}$ sample synthesized at $192{ }^{0} \mathrm{C}$ under 8.5 mbar pressure indicates the highest gas sensitivity at room temperature after 10 minutes. This implies that the smaller particle sizes always provide higher $\mathrm{CO}_{2}$ gas sensitivity due to their larger surface areas. According to the crystallite sizes estimated using Scherrer formula, these $\mathrm{CuO}$ films favor perpendicular orientation at higher sputtering pressures. The long $\mathrm{C}$-axis of $\mathrm{CuO}$ crystal cell and the higher deposition rate are responsible for this perpendicular orientation. After the gas sensitivity reached its saturated value at room temperature, the $\mathrm{CO}_{2}$ gas sensitivity was measured with temperature. The $\mathrm{CO}_{2}$ gas sensitivity of the $\mathrm{CuO}$ film fabricated at $192{ }^{0} \mathrm{C}$ and 9 mbar indicates a sudden change at operating temperature of $75{ }^{0} \mathrm{C}$. The gas sensitivity of the samples was measured in $\mathrm{N}_{2}$ gas to study the cross-sensitivity, and the sensitivity in $\mathrm{N}_{2}$ gas indicates a slight variation with time and temperature. $\mathrm{CO}_{2}$ gas sensitivity of samples sputtered at 9, 8.5, 7.5 and 6 mbar pressures reaches 3.49, 19.26, 1.55 and 1.56 , respectively after 10 minutes. 


\section{ACKNOWLEDGEMENTS}

We specially want to thank Professor R. M. G. Rajapakse and Mr. M. V. K. Perera at Department of Chemistry, University of Peradeniya for measuring XRD patterns of these samples.

\section{REFERENCES}

1. Zheng Jiao, Feng Chen, Run Su, Xingjiu Huang, Wei Liu and Jinhuai Liu, Study on the characteristics of Ag doped CuO-BaTiO sensors, Sensors 2, (2002) 366.

2. Arijit Chowdhuri, Vinay Gupta and K. Sreenivas, Thickness dependence effects of $\mathrm{CuO}$ Island on $\mathrm{SnO}_{2}$ in the nano-scale range for $\mathrm{H}_{2} \mathrm{~S}$ sensing applications, Rev. Adv. Mater. Sci. 4, (2003) 75.

3. Jinhuai Liu, Xingjiu Huang, GangYe, Wei Liu, Zheng Jiao, Wanglian Chao, Zhongbai Zhou and Zengliang $\mathrm{Yu}, \mathrm{H}_{2} \mathrm{~S}$ detection sensing characteristic of $\mathrm{CuO} / \mathrm{SnO}_{2}$ sensor, Sensors 2 , (2003) 110.

4. Wenzhong Wang, Oomman K. Varghese, Chuanmin Ruan, Maggie Paulose and Craig A. Grimes, Synthesis of $\mathrm{CuO}$ and $\mathrm{Cu}_{2} \mathrm{O}$ crystalline nanowires using $\mathrm{Cu}(\mathrm{OH})_{2}$ nanowire templates, J. Mater. Res. 18(12), (2003) 2756.

5. K. Kinoshita and T. Yamada, A new copper oxide superconductor containing carbon, Nature 357, (1992) 313.

6. Necmi Serin, Tulay Serin, Seyda Horzum and Yasemin Celik, Annealing effects on the properties of copper oxide thin films prepared by chemical deposition method, Semicond. Sci. Technol. 20, (2005) 398.

7. Andrei Catana, Jean-Pierre Locquet, Sun M. Paik and Ivan K. Schuller, Local epitaxial growth of CuO films on MgO, Phys. Rev. B46, (1992) 15477.

8. Lidia Armelao, Davide Barreca, Manuel Bertapelle, Gregorio Bottaro and Cinzia Sada and Eugenio Tondello, Sol-gel synthesis and characterization of CuO-based nanosystems, Mat. Res. Soc. Symp. Proc. Vol. 737, (2003) 27.

9. P. Samarasekara, A.G.K. Nisantha and A.S. Disanayake, High-photo voltage zinc oxide thin films deposited by DC sputtering, Chinese Journal of Physics 40(2), (2002) 196.

10.P. Samarasekara, M. A. K. Mallika Arachchi, A. S. Abeydeera, C. A. N. Fernando, A. S. Disanayake, and R.M.G. Rajapakse, Photocurrent enhancement of d.c. sputtered copper oxide thin films, Bull. Mater. Sci. 28(5), (2005) 483.

11.P. Samarasekara and F.J. Cadieu, Magnetic and structural properties of RF sputtered polycrystalline lithium mixed ferrimagnetic films, Chinese Journal of Physics 39(6), (2001) 635.

12.P. Samarasekara and F.J. Cadieu, Polycrystalline Ni ferrite films deposited by RF sputtering techniques, Jpn. J. Appl. Phys. 40, (2001) 3176.

13. F. J. Cadieu, Physics of thin films, (Academic press, San Diego 1992), Vol.16, p. 53. 